\title{
Research-Action-Research Towards a Circular Urban System for Multi-level Regeneration in Historical Cities: The Case of Bologna
}

\author{
Valentina Gianfrate, Amir Djalali, Beatrice Turillazzi, Saveria O.M. Boulanger, Martina Massari*
}

Architecture Department, University of Bologna, Bologna 40126, Italy

Corresponding Author Email: m.massari@unibo.it

https://doi.org/10.18280/ijdne.150102

Received: 8 July 2019

Accepted: 11 September 2019

\section{Keywords:}

co-design, cultural heritage, researchaction, sustainability, urban research methodology, urban planning

\begin{abstract}
Urban regeneration is a key topic in Europe as cities are asked to propose efficient actions for the transition toward a more liveable system. The singularities and specificities of each city urges for a flexible and multi-scale approach able to face and combine a mix of cultural heritage, social and economic constraints, climatic and architectural specificities. In order to work toward the definition of site-specific and flexible methods, the ROCK project investigates how to move towards a Circular Urban System Model to be applied to historic centres. The paper presents the concept and the pilot actions undertaken in Bologna to build a site-specific approach enabling local stakeholders to collaborate toward the definition of action plans for the transition into sustainable systems of places. The project adopts a multilevel methodology to create links among key areas, resources, stakeholders and tools in order to re-circulate local values for their valorisation and enhancement. The paper describes the elaboration and the research-action-research initiatives as results of Bologna's University area experimentations, deepening the relation among public spaces, local stakeholders and social exchanges. In particular, it presents the first experimentations of the project into this area: The Living Lab approach and co-design experiences.
\end{abstract}

\section{INTRODUCTION}

Urban regeneration for sustainable development is constantly under development and evolution, whose boundaries of application are wide and varied. Nevertheless, the potentialities given by the study of new ways for regenerating urban areas, considering the innovative potentials given by cultural heritage as well as by sustainable and more equitable strategies $[1,2]$, still need clear investigation and experimentation. As defined by the EU [3-5], cities are the cornerstones of European wealth, in terms of economy, welfare and future opportunities. The growth and densification of the population is, in fact, increasing year-by-year, leading to the exacerbation of challenges related to sustainability and urban management. Then, waste management, energetic demand peak, traffic congestion, air and water pollution, lack of identity, poverty fuel, are just some of the main problems on a long-term perspective [6-8].

In this context, culture and cultural heritage can be of wide support to the urban regeneration process. According to the UNESCO reports of 2016 and 2017 in fact, culture is seen as a key resource for sustainable urban development. Furthermore, the Agenda 2030, with the Goal 11 dedicated to Sustainable Cities and Communities, includes culture and creativity among the key driver to reach the goal. This inclusion confirms the role of culture in developing more sustainable cities. Preserving urban quality, protecting urban identities, valuing local cultures, both old and new, promoting cultural expressions are the pillars for making cities more sustainable, more vibrant, more liveable.

This paper analyses the research-action-research strategy developed within the Horizon 2020 project ROCK -
Regeneration and Optimisation of Cultural Heritage in creative and Knowledge cities, addressing the abovementioned need for a more comprehensive approach to understand cultural heritage's latent possibilities to develop urban innovation. In particular, in the city of Bologna such a methodology was tested through a series of participatory consultations and small-scale urban interventions.

\section{METHODOLOGY}

The ROCK project is coordinated by the Municipality of Bologna and the University of Bologna as its key scientific partner. Since 2017, ROCK proposes an experimental approach to urban regeneration for sustainable development, focusing on public space and cultural heritage as engines of innovation. The aim of the project is to support cities in tackling current and future challenges-including the reduction of their carbon footprint and the answer to unsustainable trends in mobility, education, health, food and entertainment-while providing innovative solutions to manage the impact of global phenomena. The hypothesis is that cultural heritage is a key starting point for the construction of a socio-technical infrastructure able to sustain and implement the action necessary to reach the transition towards sustainable urban regeneration. The objective of ROCK is to support the transformation of areas located in the historical centres of Bologna, Lisbon and Skopje, into creative and sustainable districts. Each city works on specific pilot areas, implementing innovative governance methods, temporary project and technological tools to monitor this transformation. The project aims to produce and transfer the knowledge 
emerged through participatory and co-design experiences to the current urban policies of the cities.

ROCK's approach considers cultural heritage as a "strategic living asset that evolves through our engagement with it" [9], a key driver for sustainable growth in European cities. Drawing from the UNESCO reports $[1,2]$ and to the Faro Convention (2005) [10] it is considered as a valuable resource for supporting mitigation and adaptation to climate change. ROCK's approach calls for overcoming a specialist idea of cultural heritage intended exclusively in terms of preservation. Cultural heritage can be seen, instead, as a growing and changing repository of processes of value sharing that can guide both spatial and behavioural change. The ROCK approach assumes the historical parts of the city, and in particular their underused spaces, as rich basins of dynamic assets to be activated and introduced into a continuous development cycle, interacting with different systems [11] This reactivation takes place through incremental experiments starting from the small scale with pop-up architectural interventions, small installations, ethnographic and cartographic observations and widespread co-design activities of the services. These punctual actions interact with geographical, administrative, ecological and social systems, generating a complexity that must be managed by a strong circular governance structure.

Therefore, the circularity of the ROCK approach can be declined in two ways: on the one hand, the establishment of the elements for a new model for sustainable urban regeneration, implies the connection of "systems" that were initially separated [11], by enabling technical, organizational and administrative solutions, but also informal and spontaneous inputs. On the other hand, circularity is based on a continuous feedback loop between cognitive practices, administrative action and reflection, shaping a researchaction-research methodology, which also proposes a new model for the collaboration between universities and public administrations. The exchange of knowledge produced, aims to generate a shared value system [12] among researchers, civil servants and the ecosystem of local stakeholders and takes place thanks to the opportunity to test the principles developed in the field of research and consolidate them through practice, to define the first steps for a new for heritageled urban regeneration.

The difficulty is to determine how these multiple sociotechnical systems can interact and produce new knowledge together, how their institutions should be adapted, and how such processes should be coordinated and facilitated. The methodology of the ROCK project, based on a continuous research-action-research feedback loop, connects and mobilises sub-systems of actors, processes and technologies in several realms. In this way, ROCK aims at redefining culture as a "living engine" to create new knowledge pathways to rethink the evolution of historic cities to prepare them for future challenges. Temporary, collective actions are implemented to re-design and increase the usability and sustainability of heritage spaces, encouraging virtuous flows within the system and creating the conditions to ensure safer, healthier and more suitable places for the communities to live and work. In the perspective of the ROCK Circular Urban System, cultural heritage is maintained and reinforced, and progressively enhanced with the addition of new components that develop on the old ones, while attracting new resources and partnerships [13]. Cultural heritage thus becomes, on the one hand, the legacy of the ability of historical centres to combine different types of knowledge to transform and adapt to contingent dynamics. On the other hand, it has the potential to become the testing ground and the observatory of a series of actions that combine different elements and systems to constantly renew itself towards the future.

\subsection{The action-research paradigm}

Within this framework, the project proposes a researchaction-research approach. This approach is neither an inductive, empirical model-going from the particular to the general, from practice to theory - nor a deductive approachwhich posits the primacy of the speculative moment and sees action as an application of a theoretical model. On the contrary, research-action finds itself always in the midst of a continuous movement, in which speculation and practice, knowledge and action can never be separated [14]

ROCK's research-action-research model draws from the current debate on the democratisation of science and the new role of research in society [15]. The need for an active engagement of citizens in research, as well as the call for an active engagement of researchers in society, was firstly felt in the social sciences. However, the widespread disaffection towards the practices of post-war urban planning and architecture forced designers and urban planners to invent new forms of pluralistic and participatory practice [16]. At the same time, citizen science, defined as the "non-professional involvement of volunteers in the scientific process, whether in the data collection phase or in other phases of the research" and participation are today necessary conditions for EU research funding in al scientific domains, as well as cornerstones for responsible research and innovation [17]. Research-action aims to put into practice the concept of citizen science, to bridge the existing gap between the theoretical dimension of learning and real-life challenges, by facilitating urban education and research, while operatively providing solutions for the city.

Drawing from the widespread debate on active learning, experiential learning, practice-oriented approaches, ROCK's proposed methodology has the ambition to generalise these models beyond a simple idea of participation in urban planning and design, as well as beyond specific disciplinary boundaries. ROCK works towards the definition of research-action as comprehensive methodology for the analysis, management and transformation of historic areas as commons.

Within ROCK, knowledge is produced as a form of collaboration among international partners as well as local inhabitants and stakeholders. On a European scale, ROCK is based on a role-model/replicator paradigm, based on international mentoring, workshops and city clusters that define the main common strategies and the methodology of evaluation, through the definition of shared key performance indicators (KPIs). On a local level, ROCK is based on a living lab approach, which supports the definition of the main priorities on the experimental sites emerged by the communities, and the guide criteria for the transformation of the areas, co-designed with them. This step is necessary for identifying not only the specific actions to be implemented, but also to map existing knowledges, instruments, tools and practices, and to complement them with the new instruments developed by the project.

Pilot actions - small-scale experimentations, prototypes of services, events, temporary urban transformations - are then deployed according to the first drafting of implementation 
scenarios and to the outcomes of the living labs. During this phase, new needs and desires, as well as the presence of unforeseen barriers, emerged, together with the tactical necessity of modifying the action plan already envisioned. Therefore, the process implies a constant need for reflection upon the results already achieved. The results are then monitored through the evaluation framework which had been previously agreed upon. In turn, the outcome of the evaluation phase becomes a new input for the management of the local cultural assets and the development of future action plans.

In this way, this approach merges bottom-up and top-down approaches that involve different levels of stakeholders and community. Research results in a more precise and needrelated scenario modelling, which includes not only foreseen actions and the application of new tools, but also considerations about new assumptions to be taken into account, new barriers and risks, new stakeholders, the connection of specific action with more precisely identified target groups, the clustering with existing events, tools and plans. This research-action-research process, between design and prototyping, could mean for urban institutions to be able to experiment small, temporary, self-concluded sequence of actions to address complex problems, before proceeding towards long-term programming and planning.

\subsection{The ROCK circular urban system}

The ROCK circular urban methodology-the ROCK Circle - has the aim of supporting cities towards the transition to sustainable urban regeneration by identifying, interrogating and assessing actions and methodologies able to create new cultural values starting from the existing ones. The application of the methodology is meant at the urban scale, nevertheless ROCK proposes an incremental approach starting trough pilot actions at the district or neighbourhood scale. The ROCK Circle, in fact, aims to turn the actions into process and to support the transformation of the historic city centre into a clustered system. For this reason, the project foresees the creation of a Creative and Sustainable District, an experimentation ground with an intermediate dimension useful for implementing projects and monitoring results effectively; the district is also recognisable by its citizens in terms of identity and sense of belonging, recognizing a geographical, a social, an administrative and a functional dimension.

The geographical limitation of this strategy's application is useful for testing solutions in a limited environment, characterized by physical boundaries. Of course, this may not be auto-conclusive, but it needs to lead to a replication and upscaling phase. In the case of ROCK demonstration areas, the boundaries of the geographical dimension are variable in relation to the issues taking into account, and to the specific experimentations carried out during the project (new routes, new connections, new services). The local geographical dimension is related to the international one, to create a comparative structure to validate the methodology, the approach, the tools, etc.

The social dimension refers to the presence of a particular and homogeneous community, or conversely an inhomogeneous community with-or without-social constraints, characterized by social conflicts, specific needs, etc. ROCK, thanks to a systemic and integrated analysis (by sensors, by field works, by Living Lab approach) maps the different social dimensions of the experimental areas, using them as a starting point to define specific solutions at urban scale.

The comprehension of the administrative dimension of the district is helpful to propose policy implementation, to understand the issues for city-branding, to recognising immediately some specific features, policies and laws that can be applied to the selected district.

Finally, ROCK has the final aim to integrate new functions and to attract new audiences in its demonstration area, so the first step is a complete recognition of the current functions, a study on the possibility to integrate the existing and the new uses, creating a balance between conservation imperatives and the need for future developments.

Creating a circular urban model implies connecting "systems" that were initially separated through not only technical but also organizational and institutional solutions and changes ("multiple innovation processes"), to be adopted and adapted. It is very important in the regeneration processes to overcome a widespread silos-thinking approach and to connect the different systems involved or related to these processes [6]. ROCK starts from identifying an ecosystem of actors and stakeholders, a system of places, a system of initiatives and resources which are already present in the city to put them together into a systemic and comprehensive vision, and works to determine how multiple socio-technical systems can interact and evolve together, how institutions and policy solutions should be adapted to the promoted circular approach, and how regeneration processes should be coordinated and facilitated (Figure 1).

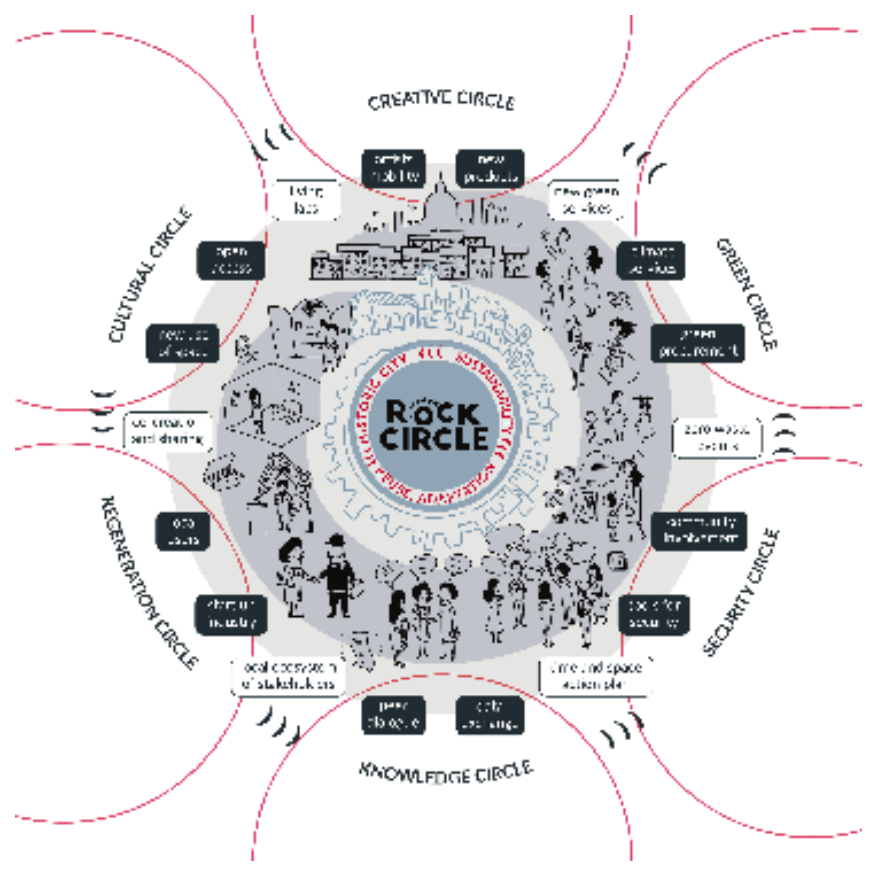

Figure 1. The ROCK Circle. Artwork Margherita Ascari, Zhai Dewei

\section{METHODOLOGY}

\subsection{The living lab and the construction of the action requirements}

In Bologna, the project works on the university area, considered an experimental site for the creation of the circular urban system model of regeneration, through spatial, 
sociological and technological solutions. The University Area (Zona-U) of Bologna is intended as a situated laboratory, where the elements used are the cultural and creative practices, aimed at producing an original scenario of development of the area, though heritage-led regeneration. Citizens and institutions, established and new actors contribute in a horizontal way to the collective co-production of urban experiments, enhancing the tangible and intangible cultural heritage. U-lab is the "vehicle" provided by the ROCK project and its partners to build knowledge from the experimentation area, to co-design priorities and requirements for its development and to plan detailed activities to be aggregated into the city schemes. It is framed as a transversal activity to the project, linking the localized experimentations in different spaces, public, private and collective, held together by the dimension of "platform".

The distribution of the actions in the area was meant to respond to the twofold objective of highlighting the presence of a widespread and underused building stock and to ensure a dynamic of ideas along the area. U-lab's attempt is to broaden the typical governance structures of the Living Lab $[18,19]$ by including the local community and actors not only in the activities but also in the definition of the action plan and management of the district agenda. The further challenge is to involve the categories that do not normally participate in the care and development of the city, such as the student population and the cultural and creative industries, disadvantaged people, with the aim of ensuring the accessibility of cultural heritage (formal and informal) to those who gravitate around the area. U-lab acts in a laboratory logic, managing different ingredients and intercepting multiple resources, building networks and defining collaborations, broad partnerships, co-planning methods to achieve a series of main objectives:

(1). define a set of shared priorities for the area, opening opportunities for meeting and exchange and helping to strengthen the agency of actors who enjoy, live, work in the area;

(2). build an ecosystem of stakeholders with whom to collectively promote a series of activities for the territory. All actions are therefore aimed at facilitating development and consolidating relationships at various levels; provide different development scenarios on which to direct the planning tools and propose alternatives;

(3). experimenting with unconventional uses of public spaces.

The activities of U-lab and U-Atelier were carried out in two stages during 2018 and 2019. The first moment is defined as "listening and co-design" and has put in place a series of exploratory activities aimed at building knowledge and priorities for action on the area; the second moment has concerned the "experimentation and prototyping" of the proposals that emerged from the co-design (Figure 2).

The first phase began with the involvement of local actors (institutional and non-institutional) to be involved in the definition of laboratory activities and in the implementation of actions. The experimentation phase has seen the materialization of the defined actions, developed through coconstruction workshops of temporary spatial arrangements and a call for ideas, through a series of events and activities carried out during the summer season, by the actors previously involved, in this phase, becoming agents of the transformation of the portion of the city.

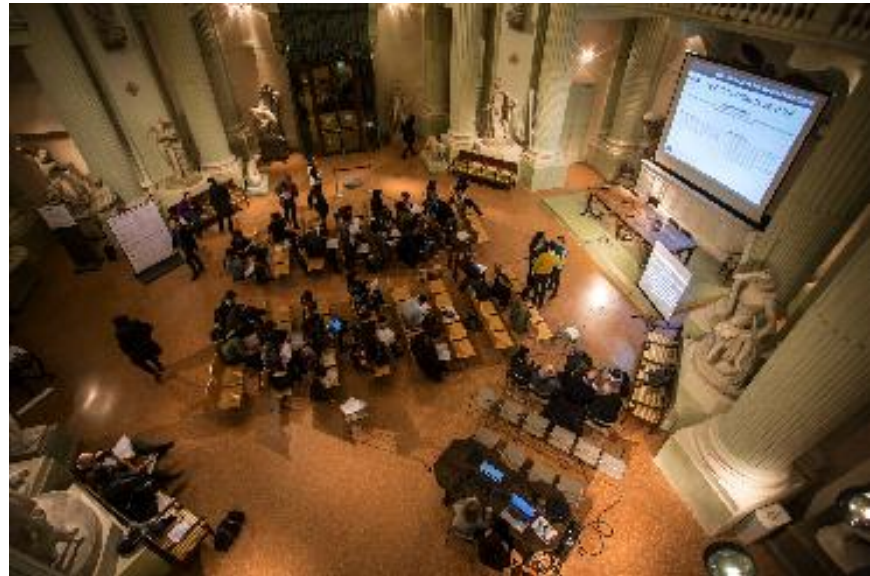

Figure 2. ROCK U-lab activities of listening and codesigning. Photo Margherita Caprilli

The first phase included seven thematic meetings on what have been selected as the three main scenarios for the regeneration of the Zona-U: sustainability, accessibility and collaboration for new cultural productions. During the meetings, which were attended by delegations of local citizen's associations, cultural producers, civil servants from local administration offices, representatives of social enterprises as well as from individual inhabitants of the area, a preliminary definition of these three concepts were presented and discussed. This allowed to get a situated idea of what sustainability, accessibility and collaboration really meant for the inhabitants and for the subjectivities operating on the university district. For instance, the issue of sustainability was more perceived in terms of the need of a collaborative effort for the care of public spaces, rather than reducing the carbon footprint and waste reduction in the area. Similarly, accessibility, rather than a simple technical matter for the reduction of physical barriers for the access to heritage buildings, was understood as a social issue, as the need for the removal of all the cultural, economic and social barriers for the access to culture. Beyond the need to help subjects that are perceived as "vulnerable" accessibility entailed for the participants to develop a community-based approach to find better solution to benefit every permanent or temporary inhabitant of the area. In an analogous way, improving collaboration for the production of culture was perceived as the need to broaden the accessibility to underused spaces to allow the participation of a broader part of the population in the organisation of events and cultural activities.

The outcome of the first phase was the development of a list of requirements for the three scenarios, which has been taken as a starting point for the deployment of actions in the university district, as well as a blueprint for the monitoring and evaluation of the same actions. At the end of the first part of U-lab's activities, the participants reflected on the activities carried out, evaluating their experience on the basis of the impacts produced, the feedback received and the difficulties encountered. The constant monitoring phase is a significant part of the process of mutual learning between institutional and non-institutional practices, which leads to the production of evidence both of research and useful to the narrative of project activities.

The outcomes of the first phase of U-Lab were taken as an input for a two-fold strategy. Firstly, a public call for project was launched to finance a series of activities that would have put in practice the approach and the guidelines elaborated 
during the meetings. Among the forty-seven proposals received, sixteen projects were financed. Projects included theatre laboratories, events, public discussions, presentations and collaborative mapping activities, which constituted not only applications of the guidelines developed in the first phase, but also their verification, as well as new occasions to gather onsite data and insights.

From the knowledge base produced in the thematic meetings and during the activities on the university area, the topic of accessibility emerged as the most urgent. In March 2019, a new call was launched, with the aim of designing and prototyping a service to be tested and then implemented for the accessibility of area.

\subsection{Piazza Scaravilli and the co-design approach}

Parallel to the living lab sessions, another intervention strategy has been elaborated for the reclamation and the activation of underused and scarcely accessible spaces in the University district. This approach was directed towards light and reversible interventions. Beyond the strategy of urban regeneration through cultural events, ROCK had the initial aim to construct communities able to think, design, realize and take care of interventions in the long term. For this reason, the design of ROCK's transformations took place during codesign workshops with inhabitants, students from various backgrounds, as well as local practitioners and designers. Whenever possible, the actual interventions are realised through simple techniques that allow a direct involvement of unskilled individuals in the construction site. In this way, codesign, self-building and collective management become key moment for the construction of a community of intents and practices able to take care of the university area and its public spaces.

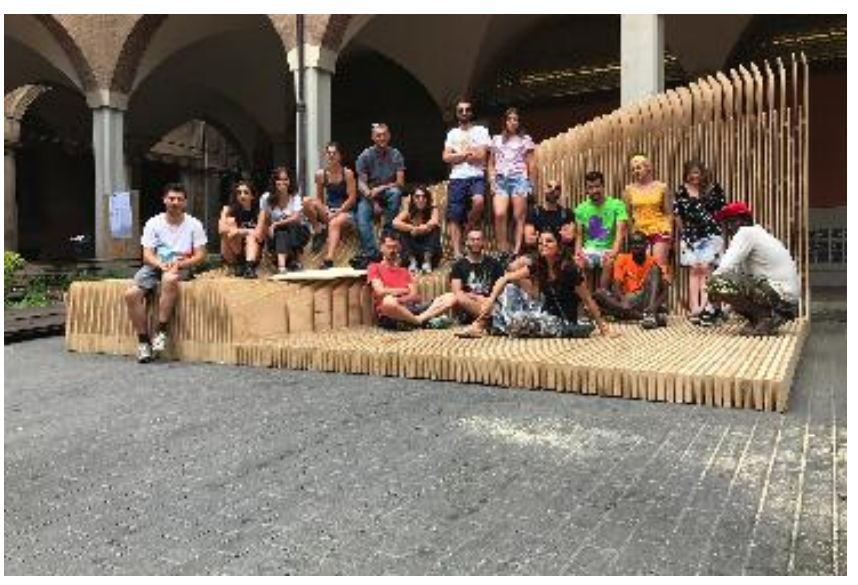

Figure 3. ROCK explores the possibility of directly involving citizens in transformation processes, allowing the exchange of value and knowledge within social ecosystems. The construction of SLAB, 6 July 2018. Photo BAG Studio

One particular place, Piazza Scaravilli, has been chosen as a testing ground for this type of experimentation. The space, a $24 \times 36$ metre square with porticoes on all sides, has been constructed in the early Fifties as part of the new faculty of Economics, on the ground left empty by Allied bombings during the Second World War. Despite its form might recall a porched piazza or a cloister, Piazza Scaravilli is rather a widened transit space. Its former use as a parking lot for the school of Economics confirms that the space has traditionally been seen as a space for mobility, and not for socialisation. However, its particular juridical status - the property is from the University, but the space of the square and the porticoes is subject to public use and therefore managed by the Municipality-as well as its modern construction-the building is not listed by the monument protection authorityallowed to select it as a fertile testing ground for the ROCK project's methodological premises.

In June 2017, a first design charrette was held with students of the department of Architecture at the University of Bologna, under the supervision of professors and researchers from the same department and the Centro Antartide, an environmental organisation active in educational activities around ecology and urban agriculture in the city. The charrette was meant to transform the space from a parking lot to a gathering place for the student community.

The design - which was self-constituted using a carpet of "pixels" made by recovered wooden fruit boxes, either filled in with ground and plants or covered to be used as seats-was built in less than one week and presented during the kick-off meeting of the ROCK project. The plant species for the installation were selected among the local spontaneous flora. Usually considered as infesting weeds, these plants are generally more resistant than traditional ornamental species. This choice oriented also the participants to call the installation Malerbe (weeds), turning a practical solution to a more symbolic function.

Malerbe was thought as a provisional and reversible intervention, as a tentative experiment not only towards new forms of use of public space, but also of the collaboration between administrations, local actors, students, inhabitants and the cultural producers of the area. The installation was the object of critiques and it spurred some conflicts - for instance, the resistance of the employees of the School of Economics for the removal of parking space, as well as the general scepticism against the shabby appearance of the low-tech materials employed. However, Malerbe was also successful in signalling the possibility of new ways of collaboration among various departments of the municipality, the Teatro Comunale - the opera house, which provided a series of summer concerts-, local association as well as the asylumseeker's protection service. In particular, Malerbe was well received by the student population, who started using the space at every hour of the day and the night. The heavy use of the spaces produced a physiological wear of the materials, which were not meant to be kept for such a long time (the installation lasted almost two years), and complaints were reported for the dirtiness of the spaces. However, despite the need for cleaning and maintenance, the installation was not the object of vandalism or improper uses. Given the general success of the initiative, in January 2018 a second workshop was held at the Teatro Comunale, titled Utopia Concreta, the workshop was attended by students from the departments of Architecture, Sociology, Agricultural sciences, with the organisational and technical support of local foundations, architectural offices and Viabizzuno, an international lighting company. Starting from the experience of Malerbe, the participants designed a semi-permanent solution for piazza Scaravilli employing more durable materials, but keeping the non-invasiveness and reversibility of the installation as the general principles of the intervention. The new installation-Malerbe Plug-in-is made by a single, 16×24 m raised platform, which allows plugging in of various kinds of equipment such as plant vases, lighting elements, seats, loudspeakers, etc. During the 
workshop, an artistic installation (SLAB) was designed in collaboration with BAG Studio, an architectural office based in Rome, and it was self-built by the students in July 2019 (Figure 3). With the idea of replicating and amplifying the methodologies experimented in Piazza Scaravilli, in March 2019 a new workshop was held to design a set of strategies to be employed in the other squares of the University district. Again, these solutions - which include the removal of parking spaces in Piazza Rossini, the test of a new signposting and wayfinding system, as well as an integration between the museums of the area and their adjacent public spaces - will be tested in a summer event that will be held in September 2019.

\section{CONCLUSION}

The experiences of U-Lab and the co-design experiments in Piazza Scaravilli were two of the main applications of ROCK's research-action-research methodology oriented towards the urban regeneration of the historic part of a city, in a limited and controlled environment. This experiment was successful in terms of allowing alternative types of interventions in historical contexts beyond usual reactivation strategy employed by the local administration based on cultural or recreational events. Also, unprecedent and innovative themes were introduced in the public debate. In particular, an original point of view on accessibility, based on the engagement of cultural institutions, businesses and individuals, is likely to produce a shift in the traditional ways in which issues of accessibility are usually tackled, paving the way for possible direction for institutional urban regeneration projects.

Co-designed temporary urban installations were successfully embraced by the local population, in particular by the students of the University of Bologna, who appropriated the space of Piazza Scaravilli with their constant presence throughout the day and the night. The small-scale, temporary interventions contributed to raise awareness on specific needs of residents and city users, and shed light on possible solutions to address them through an original valorisation of local heritage. However, the engagement device of the living lab proved insufficient to meet the active participation of who normally do not participate, especially students, In this sense, new forms of engagement should be thought beyond the living lab model, which was able to mobilise only those individuals and stakeholders already engaged in on-going formal or informal civic processes.

ROCK's research-action-research extends the principles of citizen science to the domains of urban management and design, providing a space for experimentation and mutual learning, especially concerning issues that top-down approaches are no longer sufficient to meet. ROCK's researchaction extends participation not only in the phases of the knowledge building and design, but also, through the collaborative definition of impact indicators and monitoring instruments, in the evaluation phases, providing insights for new strategies and policy implementation.

The research-action-research method, as experimented within ROCK, presents a new model for the collaboration of the university and the municipality. The main idea is to move beyond the traditional role of the university researcher as a city council's consultant, working under public commission, to produce a knowledge base for actions that would be decided behind closed doors. In ROCK, the researcher has a protagonist role both in the definition of research questions, methodologies, as well as the intervention strategies, in a continuous feedback loop. In this way, the researcher becomes an active agent in urban transformation, while city administration becomes an investigative process, and not a mere application of political decisions.

Action research has proven successful in the management of a small area, with a limited thematic focus, for a specific moment in time. In this context, it has effectively set in motion certain circular processes in terms of research, action, and it has allowed several institutions and actors to join forces and to act in common. The attempt of extending this model to other contexts, as well as to a larger scale, is however still to be verified. The success of ROCK's circular urban system method, will be the test for the generalisation of the researchaction-research paradigm and its sustainability in the long term and at a larger scale.

\section{ACKNOWLEDGMENT}

The research described in this paper is part of the outcomes of the ROCK project (https://rockproject.eu/), co-financed by the European Union within the H2020 framework programme under grant agreement no. 730280 .

\section{REFERENCES}

[1] UNESCO. (2016). Culture Urban Future. Global Report on Culture for Sustainable Urban Development, 2016. https://unesdoc.unesco.org/ark:/48223/pf0000245999, accessed on 2 July 2019.

[2] UNESCO. (2017). Re-Shaping cultural policies. Advancing creativity for development, Paris, 2017. https://unesdoc.unesco.org/ark:/48223/pf0000260678, accessed on 2 July 2019.

[3] ESPON. (2012). Territorial Impact Assessment of Policies and EU Directives. A Practical Guidance for Policymakers and Practitioners Based on Contribution from ESPON Projects and the European Commission, ESPON: $\quad$ Luxembourg, 2012. https://www.espon.eu/sites/default/files/attachments/TI A_Printed_version.pdf, accessed on 2 July 2019.

[4] European Commission. (2015). Directorate-General for Research and Innovation, State of the Innovation Union 2015, Luxembourg. https://doi.org/10.2777/805999, accessed on 2 July 2019.

[5] European Environment Agency. (2017). Trends and projections in Europe. Tracking progress towards Europe's climate and energy targets, 2017. https://www.eea.europa.eu/themes/climate/trends-andprojections-in-europe/trends-and-projections-in-europe, accessed on 2 July 2019.

[6] Boeri, A., Gaspari, J., Gianfrate, V., Longo, D., Boulanger, S.O.M. (2019). Circular city: A methodological approach for sustainable districts and Communities. WIT Transactions on The Built Environment, 183: 73-82. https://doi.org/10.2495/ARC180071

[7] Stocker, T.F., Qin, D., Plattner, G.K., Tignor, M., Allen, S.K., Boschung, J., Nauels, A., Xia, Y., Bex, V., Midgley, P.M. (2013). Climate Change 2013: The Physical Science Basis. Contribution of Working Group I to the 
Fifth Assessment Report of the Intergovernmental Panel on Climate Change, Cambridge University Press: Cambridge, UK and New York.

[8] McBean, G. (2012). Climate change: Global risks, challenges and decisions. Eos Transactions American Geophysical Union, 93(18). http://doi.org/10.1029/2012EO180011

[9] Court, S., Wijesuriya, G. (2015). People-centred approaches to the conservation of cultural heritage: Living heritage. International Centre for the Study of the Preservation and Restoration of Cultural Property, Via di San Michele, 1300153 Rome, Italy.

[10] Council of Europe Framework Convention on the Value of Cultural Heritage for Society. Council of Europe Treaty Series - No. 199. https://rm.coe.int/1680083746.

[11] Vernay, A.B.H., Singh, R. (2012). Circular urban systems-tracing innovation processes. Proceedings of the 2nd World Sustain Forum, pp. 1-30.

[12] Sennett, R. (2017). The open city. in In the Post-Urban World, edited by Haas, T., Westlund, H., Routledge: Abingdon-on-Thames, 97-106.

[13] Boeri, A., Borsari, A., Borghi, V., Gaspari, J., Gianfrate, V., Leoni, G., Longo, D. (2018). Culture as primary political action in city governance: Three key concepts and ten policies to start with. European Journal of Creative Practices in Cities and Landscapes. https://cpcl.unibo.it/article/view/8153/7840.

[14] Stark, J.L. (2014). The potential of Deweyan-inspired action research. Education and Culture, 30(2): 87-101. https://doi.org/10.1353/eac.2014.0013

[15] European Commission. (2019). Science with and for Society. Horizon 2020. Work Programme 2018-2020.

[16] Swann, C. (2002). Action research and the practice of design. Design Issues, 18(1): 49-61. https://doi.org/10.1162/07479360252756287

[17] Peter, V., Maier, F., Mejlgaard, N., Bloch, C.W., Madsen, E.B., Griessler, E., Bührer, S. (2018). Monitoring the evolution and benefits of Responsible Research and Innovation. in Europe: Summarising Insights from the MoRRI Project-Study.

[18] Bergvall-Kåreborn, B., Eriksson, C.I., Ståhlbröst, A., e Svensson, J. (2009). A milieu for innovation: Defining living labs. In ISPIM Innovation Symposium.

[19] Franz, Y. (2015). Designing social living labs in urban research. Info, 17(4): 53-66. https://doi.org/10.1108/info-01-2015-0008 